OPEN ACCESS

Edited by:

Rodrigo Torres-Castro,

University of Chile, Chile

Reviewed by:

Peijun Li,

Shanghai University of Sport, China

Ignacio Cabrera-Aguilera,

University of Barcelona, Spain

*Correspondence:

Hailiang Huang

06000031@sdutcm.edu.cn

Specialty section:

This article was submitted to

Pulmonary Medicine,

a section of the journal

Frontiers in Medicine

Received: 10 November 2021

Accepted: 11 January 2022

Published: 02 February 2022

Citation:

Li L, Huang H, Song J, Yu Y, Jia Y, Wang $Y$, Dang $X$, Huang $L$ and $L i u X$ (2022) Network Meta-Analysis of the Effects of Different Types of Traditional

Chinese Exercises on Pulmonary

Function, Endurance Capacity and

Quality of Life in Patients With COPD.

Front. Med. 9:806025.

doi: 10.3389/fmed.2022.806025

\section{Network Meta-Analysis of the Effects of Different Types of Traditional Chinese Exercises on Pulmonary Function, Endurance Capacity and Quality of Life in Patients With COPD}

\author{
Lingling $\mathrm{Li}^{1}$, Hailiang Huang ${ }^{1 *}$, Jiao Song ${ }^{2}$, Ying $\mathrm{Yu}^{3}$, Yuqi Jia ${ }^{1}$, Yajie Wang ${ }^{1}$, \\ Xiaowen Dang ${ }^{1}$, Lei Huang ${ }^{1}$ and Xinyue Liu ${ }^{4}$ \\ ${ }^{1}$ College of Rehabilitation Medicine, Shandong University of Traditional Chinese Medicine, Jinan, China, ${ }^{2}$ College of Health, \\ Shandong University of Traditional Chinese Medicine, Jinan, China, ${ }^{3}$ Innovative Institute of Chinese Medicine and Pharmacy, \\ Shandong University of Traditional Chinese Medicine, Jinan, China, ${ }^{4}$ College of Physical Education, Hebei Normal University, \\ Shijiazhuang, China
}

Background: In recent years, Chinese and international studies have reported that traditional Chinese exercises (TCEs) have good therapeutic effects on pulmonary function, endurance capacity, and quality of life in patients with chronic obstructive pulmonary disease (COPD). However, only a few studies have reported the differences in the efficacy of different TCEs in the treatment of COPD.

Objective: The objective of this study is to compare the effects of five TCEs on patients with COPD, including Taijiquan (TJQ), Baduanjin (BDJ), Liuzijue (LZJ), Wuqinxi (WQX), and Yijinjing (YJJ).

Methods: All randomized controlled trials (RCTs) of TCEs for patients with COPD were searched in PubMed, Web of Science, Cochrane Library, Excerpt Medica Database (EMBASE), China National Knowledge Infrastructure (CNKI), China Biology Medicine database (CBM), China Scientific Journal Database (VIP), and Wanfang database. The search period was from the establishment of each database to August 16, 2021. The quality of the included studies was assessed according to the Cochrane handbook of systematic review, and the network meta-analysis was conducted with R 4.0.2 (Ross Ihaka, Auckland, New Zealand) and ADDIS 1.16.8 (Gert vsn Valkenhoef, Groningen, Netherlands). The effect size was evaluated using the mean difference (MD) and 95\% confidence interval $(\mathrm{Cl})$.

Results: A total of 53 RCTs involving 3,924 patients were included. The network meta-analysis results showed that WQX was the most effective in improving $\mathrm{FEV}_{1} / \mathrm{FVC} \%$ score and 6-MWT score. The difference was statistically significant (MD $=8.62,95 \% \mathrm{Cl}$ 4.46 to 13.04, $P<0.05$ ), (MD $=74.29,95 \% \mathrm{Cl} 47.67$ to 102.24, $P<0.05$ ). However, YJJ was the most effective in reducing the CAT score, and the difference was statistically significant (MD $=-8.38,95 \% \mathrm{Cl}-13.24$ to $-3.28, P<0.05)$.

Conclusion: The existing evidence shows that WQX has advantages over other TCEs in improving pulmonary function and endurance capacity in patients with COPD, 
while YJJ has advantages in improving the quality of life. Although TCEs show no significant adverse effects, more large-scale, double-blind, and high-quality RCTs are needed in the future to verify the findings of this study.

\section{Systematic Review Registration: https://www.crd.york.ac.uk/PROSPERO/,} identifier: CRD42021293640.

Keywords: traditional Chinese exercises, chronic obstructive pulmonary disease, pulmonary function, exercise endurance, quality of life, network meta-analysis

\section{INTRODUCTION}

Chronic obstructive pulmonary disease (COPD) is a respiratory system disease characterized by persistent airflow restriction, which develops gradually and is not completely reversible $(1,2)$. Studies have shown that the morbidity and mortality of COPD remain high all year round, making it the third leading cause of death after ischemic heart disease and stroke. Among the deaths induced by COPD, $90 \%$ occur in low-income and middleincome countries (3). Globally, the burden of COPD is expected to increase in the coming decades due to continued exposure to risk factors and the aging population (4). Nevertheless, this disease can be prevented and treated. That is to say, standardized rehabilitation for COPD patients can delay the acute exacerbation and progression of the disease, improve their quality of life, and reduce the disability and mortality rates (4).

The goal of COPD rehabilitation treatment is to improve refractory and intractable dysfunction, restore effective abdominal breathing, improve respiratory function, and increase respiratory efficiency (5). During treatment, various measures are taken to reduce and treat complications, improve pulmonary function and physical strength, and recover mobility, thus improving the quality of life, reducing hospitalization rates, and extending life expectance (5). At present, pulmonary rehabilitation is considered an important component of comprehensive care for patients with COPD. Exercise is regarded as a core element of pulmonary rehabilitation (e.g., endurance training), with the main aim of improving the aerobic capacity of patients (6). Most patients with COPD have difficulty maintaining high-intensity and sustained exercise training because of dyspnea, fatigue, and being old (6). Therefore, there is an urgent need to find a personalized exercise mode with moderate and low intensity.

The traditional Chinese exercises (TCEs) has been inherited and reformed, based on the holistic view of human life, combining Chinese medicine health care with Chinese medicine health preservation, which plays a very good role in strengthening body and preventing disease (7). In recent years, a growing number of studies have reported that TCEs can be used as an alternative therapy for pulmonary function, endurance capacity, and quality of life in patients with COPD. However, the sample sizes of single studies are small, and the inclusion criteria and methods vary from different studies. The lack of evidence-based studies on the efficacies of different TCEs in treating COPD makes it difficult to guide clinical practice.
Meta-analysis allows the comparison of the effects between two kinds of interventions. Compared with meta-analysis, the network meta-analysis has the advantage of providing a comprehensive and quantitative analysis of the relative effects of different interventions in treating the same disease. The network meta-analysis also provides a reliable, comprehensive, and robust evidence-based basis for selecting clinical treatment schemes by sorting out the advantages and disadvantages of efficacy and safety (8). The objective of this study is to compare the effects of five TCEs on COPD by network meta-analysis and probability ranking, thus providing a reference for selecting clinical treatment optimization schemes.

\section{MATERIALS AND METHODS}

This systematic review and network meta-analysis has been registered on the international system evaluation registration platform PROSPERO (NO. CRD42021293640). This study was performed according to the Cochrane Handbook of Systematic Review and followed the statement of preferred reporting item of systematic review and meta-analysis (PRISMA) for network meta-analysis (9).

\section{Data Sources and Search Strategy}

All articles on TCEs for patients with COPD were searched by two reviewers in PubMed, Web of Science, Cochrane Library, Excerpt Medica Database (EMBASE), China National Knowledge Infrastructure (CNKI), China Biology Medicine database (CBM), China Scientific Journal Database (VIP), and Wanfang database. The search period is from the establishment of each database to August 16, 2021. A combination of Mesh words and free words search method was used in each database for comprehensive searches.

Taking PubMed database as an example, the search strategy is (traditional Chinese exercises OR traditional exercise therapy OR traditional exercise OR health qigong OR qigong OR tai chi exercise OR tai chi OR taijiquan OR liuzijue OR baduanjin OR eight section brocade OR wuqinxi OR yijinjing) AND (chronic obstructive pulmonary disease OR COPD OR chronic obstructive lung disease OR chronic obstructive airway disease OR chronic airflow obstruction).

\section{Inclusion and Exclusion Criteria}

The inclusion criteria of this study was based on the five main principles of the Participant-Intervention-ComparatorOutcomes-Study design (PICOS). 


\section{Participants}

Patients were diagnosed with COPD based on the 2017 Global Initiative for COPD (10) or other similar diagnostic criteria.

\section{Interventions}

The interventions are TCEs, including Taijiquan (TJQ), Baduanjin (BDJ), Liuzijue (LZJ), Wuqinxi (WQX), and Yijinjing (YJJ).

\section{Comparator}

The comparator is non-exercise intervention.

\section{Outcome Indicators}

The outcome indicators include the ratio of forced expiratory volume in the first second to forced vital capacity (FEV1/FVC\%), the 6-min walking test (6MWT), the COPD assessment test (CAT) and adverse events.

\section{Studies}

The studies are randomized controlled trials (RCTs) of TCEs for patients with COPD. The language of studies is limited to Chinese and English.

\section{Exclusion Criteria}

The following studies should be excluded: non-RCTs; case reports; protocols; animal experiments; meeting abstracts; reviews; studies of other interventions; treatments combined with other therapeutic interventions; studies with incomplete data; studies with non-comparable or unreported baselines; studies with no results.

\section{Data Extraction}

At first, duplicate records were removed by EndNote X9 (Thomson ResearchSoft, Connecticut, USA) software. Then, two reviewers independently read the titles and abstracts for preliminary screening, followed by a re-screening of the full text that might meet the inclusion criteria. Finally, the following data were extracted from the studies meeting the inclusion criteria: the first author; year of publication; patient age; sample size; interventions; outcome indicators; quality assessment; other information.

\section{Quality Assessment of Included Studies}

The quality of included studies was assessed by two reviewers according to the Cochrane handbook for systematic reviews. If the assessment results were different, the studies were referred to a third reviewer for assessment. The assessment items included random sequence generation, allocation concealment, blind experiments, data integrity, selective reporting, and other biases. The quality assessment of the included studies was based on three options: high risk, low risk, and unclear (11).

\section{Statistical Analysis}

In this study, network meta-analysis was performed using R 4.0.2 (Ross Ihaka, Auckland, New Zealand) and ADDIS 1.16.8 (Gert vsn Valkenhoef, Groningen, Netherlands) software. FEV1/FVC\%, 6MWT, and CAT are continuous variables, and the effect value was expressed by mean difference (MD) and $95 \%$ confidence interval (CI). When the $95 \%$ CI did not contain 0 , there was a statistical difference between the two groups. Based on the Markov Chain-Monte Carlo algorithm, the network metaanalysis and probability ranking was carried out using four chains and consistency models. The initial value was set to 0.5 , the step size was 10 , and the number of iterations was 50,000 times. The first 20,000 iterations were used for annealing to eliminate the influence of the initial value, and the last 30,000 iterations were used for sampling. The potential scale reduction factor (PSRF) was calculated to evaluate convergence by comparing the variance within and between chains. A PSRF close to 1 indicated good convergence of the consistency model analysis and more reliable results (12).

\section{RESULTS}

\section{Literature Selection}

A total of 1,764 studies were obtained from the databases, and 1,149 studies were obtained after eliminating duplicate articles. Afterward, 142 studies were selected by reading the titles and abstracts. Finally, 53 RCTs (13-65) were included by reading the full-text and excluding non-RCTs, meeting abstracts, protocols, and studies with incomplete data. The flow diagram of literature selection is shown in Figure 1.

\section{Characteristics of Included Studies}

A total of 53 RCTs (13-65) with 3,924 patients were included in network meta-analysis, including 1,973 patients in the experimental group and 1,951 patients in the control group. The characteristics of the included studies are shown in Table 1.

\section{Risk of Bias Assessment}

Randomization was mentioned in 53 RCTs (13-65), 33 RCTs (13-64) described the specific methods for generating random sequences, and only 4 RCTs $(21,41,43,60)$ were related to allocation concealment. The study itself could not be blinded to researchers or patients, and 20 RCTs (13-65) were blinded to the outcome indicators. Data integrity, selective reporting, and other risks of bias are shown in Figure 2.

\section{Network Meta-Analysis}

The network diagram of TCEs for patients with COPD is shown in Figure 3. The connection between two yellow balls indicates directly comparable RCTs between the two interventions, and no connection indicates no directly comparable RCTs, which can be indirectly compared using the control group as a reference. The thicker line between the two yellow balls indicates a higher number of RCTs. Consistency analysis of FEV1/FVC\%, 6MWT, and CAT outcome indicators from 53 studies showed that all PSRF parameters were close to 1.00 , indicating good convergence of network meta-analysis. The results of the network metaanalysis and probability ranking are shown in Tables 2,3 , and Figure 4. The positive scoring outcome indicators (FEV1/FVC\% and $6 \mathrm{MWT}$ ) were ranked with the probability by Rank 1 , and higher Rank 1 represents great outcome indicators. The CAT negative scoring outcome indicators were ranked with the probability by Rank N. The higher Rank N represents great outcome indicators. 


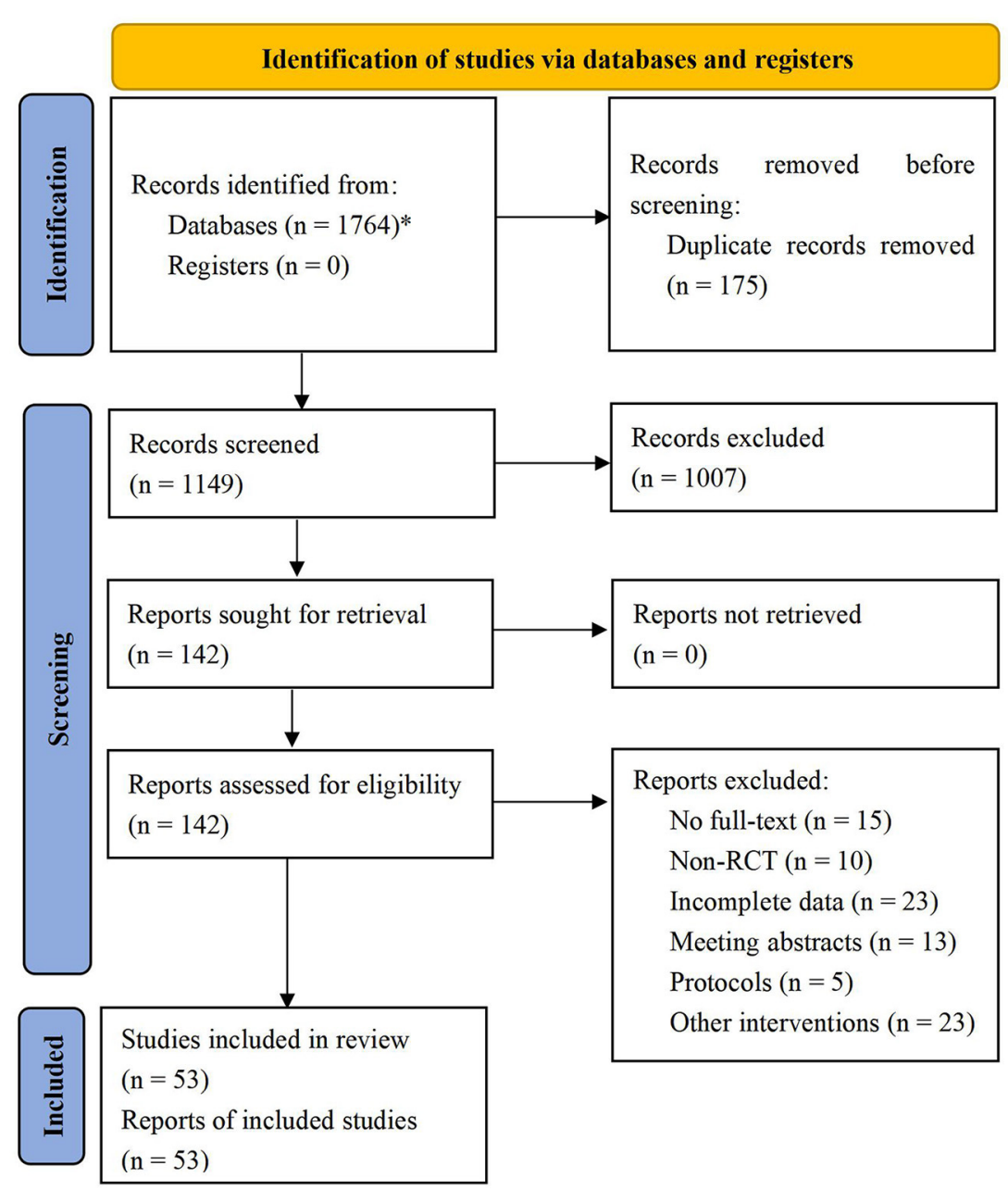

FIGURE 1 | Flow diagram of literature selection. ${ }^{*}$ PubMed $=253$, Web of Science $=247$, Cochrane Library $=95$, EMBASE $=104$, CNKI $=271$, CBM $=156$, VIP $=$ 315 , Wanfang $=323$.

\section{$\mathrm{FEV}_{1} / \mathrm{FVC} \%$}

A total of 43 RCTs (13-65) reported $\mathrm{FEV}_{1} / \mathrm{FVC} \%$ scores, involving five TCEs and 3,052 patients. The following comparisons are statistically significant: comparison of BDJ and WQX (MD $=-5.15,95 \% \mathrm{CI}-10.47$ to $-0.03, P<0.05)$; comparison of $\mathrm{BDJ}$ and the control group ( $\mathrm{MD}=3.45,95 \% \mathrm{CI}$ 0.49 to $6.44, P<0.05$ ); comparison of LZJ and the control group $(\mathrm{MD}=4.43,95 \% \mathrm{CI} 1.12$ to $7.70, P<0.05)$; comparison of TJQ and the control group $(\mathrm{MD}=4.05,95 \% \mathrm{CI} 0.71$ to $7.34, P<$ $0.05)$; comparison of $\mathrm{WQX}$ and the control group $(\mathrm{MD}=8.62$, $95 \%$ CI 4.46 to $13.04, P<0.05)$. The other comparisons were not statistically significant $(P>0.05)$. The probability ranking is: $\mathrm{WQX}(P=0.81)>\mathrm{YJJ}(P=0.12)>\mathrm{LZJ}(P=0.04)>\mathrm{TJQ}(P=$ $0.02)>\operatorname{BDJ}(P=0.01)$.

\section{MWT}

A total of 35 RCTs (15-65) reported 6MWT scores, involving five TCEs and 2,446 patients. The following comparisons are statistically significant: comparison of $\mathrm{BDJ}$ and the control group
$(\mathrm{MD}=53.47,95 \% \mathrm{CI} 36.18$ to $71.58, P<0.05)$; comparison of the LZJ and the WQX (MD $=-40.02,95 \%$ CI -76.53 to -4.10 , $P<0.05$ ); comparison of the LZJ and the control group (MD $=34.32,95 \% \mathrm{CI} 10.44$ to $58.78, P<0.05)$; comparison of TJQ and WQX (MD $=-43.68,95 \% \mathrm{CI}-78.02$ to $-9.40, P<0.05)$; comparison of TJQ and the control group ( $\mathrm{MD}=30.69,95 \% \mathrm{CI}$ 10.14 to $52.87, P<0.05)$; comparison of $\mathrm{WQX}$ and $\mathrm{YJJ}(\mathrm{MD}=$ 55.74, 95\% CI 7.58 to 105.11, $P<0.05$ ); comparison of WQX and the control group ( $\mathrm{MD}=74.29,95 \% \mathrm{CI} 47.67$ to $102.24, P<$ $0.05)$. The other comparisons were not statistically significant $(P$ $>0.05)$. The probability ranking is: $\mathrm{WQX}(P=0.88)>\mathrm{BDJ}(P=$ $0.09)>\operatorname{LZJ}(P=0.01)=\mathrm{YJJ}(P=0.01)>\mathrm{TJQ}(P=0)$.

\section{CAT}

A total of 15 RCTs $(19,21,22,26,29,38,39,44,45,47-$ $49,55,63,65$ ) reported CAT scores, involving five TCEs and 1,269 patients. The following comparisons are statistically significant: comparison of $\mathrm{BDJ}$ and $\mathrm{YJJ}(\mathrm{MD}=5.92,95 \% \mathrm{CI} 0.23$ to 11.20 , $P<0.05)$; comparison of $\mathrm{BDJ}$ and the control group $(\mathrm{MD}=$ 
TABLE 1 | Characteristics of included studies.

\begin{tabular}{|c|c|c|c|c|c|c|c|c|}
\hline \multirow[t]{2}{*}{ References } & \multirow{2}{*}{$\begin{array}{c}\text { Simple } \\
\text { Size } \\
\text { E/C }\end{array}$} & \multirow{2}{*}{$\begin{array}{l}\text { Male/ } \\
\text { Female }\end{array}$} & \multirow{2}{*}{$\begin{array}{l}\text { Age (years) } \\
\text { Mean } \pm \text { SD }\end{array}$} & \multirow{2}{*}{$\begin{array}{c}\text { Duration of } \\
\text { disease (years) }\end{array}$} & \multicolumn{2}{|c|}{ Intervention } & \multirow[t]{2}{*}{ Intervention frequency and duration } & \multirow[t]{2}{*}{ Outcomes } \\
\hline & & & & & $\mathbf{E}$ & C & & \\
\hline Deng et al. (13) & $32 / 32$ & $\begin{array}{l}31 / 1 \\
29 / 3\end{array}$ & $\begin{array}{l}66.26 \pm 5.13 / \\
66.90 \pm 4.63\end{array}$ & $\begin{array}{l}4.68 \pm 2.54 / \\
4.77 \pm 2.52\end{array}$ & BDJ & $\mathrm{NEl}$ & 30 min per time, 1 time per day for 3 months & (1) \\
\hline Liang et al. (14) & $41 / 41$ & $\mathrm{~N} / \mathrm{A}$ & $\mathrm{N} / \mathrm{A}$ & $\mathrm{N} / \mathrm{A}$ & BDJ & $\mathrm{NEI}$ & 30 min per time, 1 time per day for 3 months & (1) \\
\hline Lei et al. (15) & $49 / 50$ & $\begin{array}{l}35 / 14 \\
38 / 12\end{array}$ & $\begin{array}{l}60.45 \pm 4.76 / \\
61.78 \pm 5.32\end{array}$ & $\begin{array}{l}5.37 \pm 1.21 / \\
5.28 \pm 1.12\end{array}$ & BDJ & $\mathrm{NEI}$ & 60 min per time, 1 time per day for 12 months & (2) \\
\hline Hou et al. (16) & $25 / 23$ & $\begin{array}{l}14 / 11 \\
13 / 10\end{array}$ & $\begin{array}{l}63.34 \pm 5.95 / \\
62.87 \pm 6.01\end{array}$ & N/A & BDJ & $\mathrm{NEl}$ & $\begin{array}{l}30 \text { min per time, } 2 \text { times per week, for } 3 \\
\text { months }\end{array}$ & (1)(2) \\
\hline Wu et al. (17) & $50 / 50$ & $\begin{array}{l}26 / 24 \\
25 / 25\end{array}$ & $\begin{array}{l}62.40 \pm 5.60 / \\
60.70 \pm 6.30\end{array}$ & $\begin{array}{l}8.90 \pm 3.10 / \\
8.40 \pm 2.50\end{array}$ & BDJ & $\mathrm{NEl}$ & 30 min per time, 1 time per day for 6 months & (2) \\
\hline Guo et al. (18) & $30 / 30$ & $\begin{array}{l}13 / 17 \\
14 / 16\end{array}$ & $\mathrm{~N} / \mathrm{A}$ & $\mathrm{N} / \mathrm{A}$ & BDJ & $\mathrm{NEl}$ & $\begin{array}{l}\text { 15 20 min per time, } 1 \text { time per day for } 6 \\
\text { months }\end{array}$ & (1) \\
\hline Guo et al. (19) & $161 / 159$ & $\begin{array}{l}94 / 67 \\
96 / 63\end{array}$ & $\begin{array}{l}64.15 \pm 8.97 / \\
64.87 \pm 8.86\end{array}$ & $\begin{array}{l}16.21 \pm 5.53 / \\
16.19 \pm 5.48\end{array}$ & BDJ & $\mathrm{NEl}$ & 30 min per time, 1 time per day for 6 months & (1)(3) \\
\hline Deng et al. (20) & $27 / 27$ & $\begin{array}{l}25 / 2 \\
26 / 1\end{array}$ & $\begin{array}{l}64.84 \pm 9.03 / \\
63.92 \pm 8.47\end{array}$ & $\begin{array}{l}9.45 \pm 3.44 / \\
8.56 \pm 4.29\end{array}$ & BDJ & $\mathrm{NEl}$ & 30 min per time, 1 time per day for 6 months & (1)(2) \\
\hline Zheng et al. (21) & $19 / 19$ & $\begin{array}{l}18 / 1 \\
18 / 1\end{array}$ & $\begin{array}{l}69.89 \pm 8.89 / \\
69.68 \pm 6.84\end{array}$ & N/A & BDJ & $\mathrm{NEl}$ & $\begin{array}{l}20 \sim 30 \text { min per time, } 5 \sim 7 \text { times per week, for } \\
3 \text { months }\end{array}$ & (1)(2)(3) \\
\hline Sun et al. (22) & $40 / 40$ & $\begin{array}{l}26 / 14 \\
27 / 13\end{array}$ & $\begin{array}{l}62.97 \pm 6.87 / \\
63.21 \pm 7.02\end{array}$ & $\begin{array}{l}11.02 \pm 3.38 / \\
10.85 \pm 3.53\end{array}$ & BDJ & $\mathrm{NEl}$ & 30 min per time, 1 time per day for 12 months & (2)(3) \\
\hline Zhu et al. (23) & $63 / 60$ & $\begin{array}{l}36 / 27 \\
31 / 19\end{array}$ & $\begin{array}{l}69.00 \pm 8.70 / \\
68.00 \pm 9.20\end{array}$ & $\begin{array}{c}12.50 \pm 10.70 / \\
10.80 \pm 8.90\end{array}$ & BDJ & $\mathrm{NEl}$ & 30 min per time, 2 times per day for 6 months & (1)(2) \\
\hline Liu et al. (24) & $40 / 40$ & $\begin{array}{l}21 / 19 \\
22 / 18\end{array}$ & $\begin{array}{l}59.77 \pm 7.08 / \\
60.67 \pm 6.95\end{array}$ & N/A & BDJ & $\mathrm{NEl}$ & 30 min per time, 1 time per day for 3 months & (1)(2) \\
\hline Chen et al. (25) & $39 / 39$ & $\begin{array}{l}74 / 43 \\
73 / 42\end{array}$ & $\begin{array}{l}60.52 \pm 7.24 / \\
59.67 \pm 6.91\end{array}$ & $\mathrm{~N} / \mathrm{A}$ & BDJ & $\mathrm{NEl}$ & 30 min per time, 1 time per day for 3 months & (1) \\
\hline Huang et al. (26) & $31 / 31$ & $\begin{array}{l}20 / 11 \\
18 / 13\end{array}$ & $\begin{array}{l}68.24 \pm 3.28 / \\
69.77 \pm 4.42\end{array}$ & $\begin{array}{l}2.05 \pm 0.88 / \\
1.44 \pm 1.20\end{array}$ & BDJ & $\mathrm{NEl}$ & 30 min per time, 2 times per day for 6 months & (1)(3) \\
\hline Yu et al. (27) & $41 / 41$ & $\mathrm{~N} / \mathrm{A}$ & $\begin{array}{l}62.30 \pm 1.20 / \\
62.30 \pm 1.50\end{array}$ & $\begin{array}{l}5.30 \pm 1.50 / \\
7.30 \pm 1.40\end{array}$ & BDJ & $\mathrm{NEl}$ & 30 min per time, 1 time per day for 3 months & (2) \\
\hline Pan et al. (28) & $42 / 42$ & $\begin{array}{l}26 / 16 \\
29 / 13\end{array}$ & $\begin{array}{l}60.70 \pm 5.60 / \\
61.80 \pm 7.20\end{array}$ & $\begin{array}{l}6.70 \pm 6.20 / \\
8.80 \pm 5.30\end{array}$ & BDJ & $\mathrm{NEl}$ & 30 min per time, 1 time per day for 6 months & (1) (2) \\
\hline Dong et al. (29) & $46 / 46$ & $\begin{array}{l}27 / 19 \\
25 / 21\end{array}$ & $\begin{array}{l}63.97 \pm 5.57 / \\
64.25 \pm 6.01\end{array}$ & $\mathrm{~N} / \mathrm{A}$ & BDJ & $\mathrm{NEl}$ & 30 min per time, 1 time per day for 6 months & (2)(3) \\
\hline Wang et al. (30) & $37 / 36$ & $\begin{array}{l}27 / 10 \\
25 / 11\end{array}$ & $\begin{array}{l}63.17 \pm 9.95 / \\
63.67 \pm 9.75\end{array}$ & $\begin{array}{l}15.17 \pm 6.73 / \\
14.83 \pm 7.89\end{array}$ & BDJ & $\mathrm{NEl}$ & 30 min per time, 1 time per day for 3 months & (1) (2) \\
\hline Shi et al. (31) & $20 / 20$ & $\begin{array}{l}18 / 2 \\
15 / 5\end{array}$ & $\begin{array}{l}71.66 \pm 7.22 / \\
73.28 \pm 6.15\end{array}$ & N/A & LZJ & $\mathrm{NEl}$ & 3 months & (1) \\
\hline Zhang et al. (32) & $60 / 60$ & $\mathrm{~N} / \mathrm{A}$ & $\begin{array}{l}71.30 \pm 2.96 / \\
72.90 \pm 3.25\end{array}$ & $\mathrm{~N} / \mathrm{A}$ & LZJ & $\mathrm{NEI}$ & 30 min per time, 1 time per day for 3 months & (2) \\
\hline Zheng et al. (33) & $30 / 30$ & $\begin{array}{l}13 / 17 \\
16 / 14\end{array}$ & N/A & $\mathrm{N} / \mathrm{A}$ & LZJ & $\mathrm{NEI}$ & $\begin{array}{l}40 \text { min per time, } 5 \text { times per week, for } 6 \\
\text { months }\end{array}$ & (1) (2) \\
\hline Chen et al. (34) & $21 / 19$ & $\begin{array}{l}19 / 2 \\
14 / 5\end{array}$ & $\begin{array}{l}71.76 \pm 7.31 / \\
73.32 \pm 6.33\end{array}$ & $\mathrm{~N} / \mathrm{A}$ & LZJ & $\mathrm{NEI}$ & 3 months & (1) \\
\hline Peng et al. (35) & $30 / 30$ & $\begin{array}{l}20 / 10 \\
19 / 11\end{array}$ & $\begin{array}{l}56.22 \pm 4.17 / \\
56.17 \pm 4.12\end{array}$ & $\mathrm{~N} / \mathrm{A}$ & LZJ & $\mathrm{NEl}$ & 30 min per time, 1 time per day for 3 months & (1) \\
\hline Hou et al. (36) & $54 / 54$ & $\begin{array}{l}30 / 24 \\
29 / 25\end{array}$ & $\mathrm{~N} / \mathrm{A}$ & $\mathrm{N} / \mathrm{A}$ & LZJ & $\mathrm{NEl}$ & 6 months & (1) \\
\hline Ji et al. (37) & $28 / 29$ & $\begin{array}{c}23 / 5 \\
19 / 10\end{array}$ & $\begin{array}{l}63.75 \pm 5.48 / \\
64.52 \pm 5.68\end{array}$ & $\begin{array}{l}4.68 \pm 2.61 / \\
3.76 \pm 2.06\end{array}$ & LZJ & $\mathrm{NEl}$ & $\begin{array}{l}30 \text { min per time, } 5 \text { times per week, for } 3 \\
\text { months }\end{array}$ & (1) \\
\hline Liu et al. (38) & $27 / 25$ & $\begin{array}{l}14 / 13 \\
13 / 12\end{array}$ & $\begin{array}{l}66.15 \pm 6.43 / \\
66.40 \pm 8.84\end{array}$ & $\begin{array}{l}9.15 \pm 6.89 / \\
9.60 \pm 6.16\end{array}$ & ᄂZJ & $\mathrm{NEl}$ & 4 times per week for 6 months & (1)(3) \\
\hline Lan et al. (39) & $42 / 42$ & $\begin{array}{l}23 / 19 \\
20 / 22\end{array}$ & $\begin{array}{l}67.24 \pm 3.21 / \\
67.02 \pm 3.48\end{array}$ & $\begin{array}{c}8.29 \pm 2.76 / \\
8.21 \pm 2.83\end{array}$ & LZJ & $\mathrm{NEI}$ & 5 times per week for 3 months & (1)(3) \\
\hline
\end{tabular}


TABLE 1 | Continued

\begin{tabular}{|c|c|c|c|c|c|c|c|c|}
\hline \multirow[t]{2}{*}{ References } & \multirow{2}{*}{$\begin{array}{c}\text { Simple } \\
\text { Size } \\
\text { E/C }\end{array}$} & \multirow{2}{*}{$\begin{array}{l}\text { Male/ } \\
\text { Female }\end{array}$} & \multirow{2}{*}{$\begin{array}{l}\text { Age (years) } \\
\text { Mean } \pm \text { SD }\end{array}$} & \multirow{2}{*}{$\begin{array}{c}\text { Duration of } \\
\text { disease (years) }\end{array}$} & \multicolumn{2}{|c|}{ Intervention } & \multirow[t]{2}{*}{ Intervention frequency and duration } & \multirow[t]{2}{*}{ Outcomes } \\
\hline & & & & & $\mathbf{E}$ & C & & \\
\hline Guan et al. (40) & $31 / 32$ & $\begin{array}{l}28 / 3 \\
30 / 2\end{array}$ & $\begin{array}{l}68.52 \pm 6.29 / \\
69.56 \pm 5.13\end{array}$ & $\begin{array}{l}8.19 \pm 2.69 / \\
9.12 \pm 2.08\end{array}$ & LZJ & NEl & 30 min per time, 1 time per day for 4 months & (1)(2) \\
\hline Li et al. (41) & $17 / 19$ & $\begin{array}{l}14 / 3 \\
14 / 5\end{array}$ & $\begin{array}{l}66.00 \pm 9.00 / \\
66.00 \pm 9.00\end{array}$ & $\begin{array}{l}8.00 \pm 5.00 / \\
9.00 \pm 4.00\end{array}$ & LZJ & $\mathrm{NEl}$ & $\begin{array}{l}60 \text { min per time, } 4 \text { times per week, for } 6 \\
\text { months }\end{array}$ & (1)(2) \\
\hline Xiao et al. (42) & $63 / 63$ & $\begin{array}{l}58 / 5 \\
59 / 4\end{array}$ & $\begin{array}{l}72.20 \pm 1.70 / \\
70.90 \pm 1.40\end{array}$ & $\mathrm{~N} / \mathrm{A}$ & LZJ & $\mathrm{NEI}$ & 30 min per time, 1 time per day for 6 months & (2) \\
\hline Wu et al. (43) & $16 / 17$ & $\begin{array}{l}14 / 2 \\
14 / 3\end{array}$ & $\begin{array}{l}67.00 \pm 8.00 / \\
66.00 \pm 9.00\end{array}$ & $\begin{array}{l}13.00 \pm 4.00 / \\
12.00 \pm 4.00\end{array}$ & LZJ & $\mathrm{NEl}$ & $\begin{array}{l}40 \text { min per time, } 6 \text { times per week, for } 6 \\
\text { months }\end{array}$ & (1)(2) \\
\hline Pan et al. (44) & $20 / 21$ & $\begin{array}{l}14 / 6 \\
14 / 7\end{array}$ & N/A & $\mathrm{N} / \mathrm{A}$ & TJQ & $\mathrm{NEl}$ & $\begin{array}{l}30 \text { min per time, } 3 \text { times per week, for } 2 \\
\text { months }\end{array}$ & (2)(3) \\
\hline Zhang et al. (45) & $18 / 18$ & $\begin{array}{l}14 / 4 \\
12 / 6\end{array}$ & $\begin{array}{l}68.02 \pm 6.91 / \\
66.71 \pm 5.84\end{array}$ & $\begin{array}{l}33.41 \pm 2.45 / \\
32.84 \pm 1.98\end{array}$ & TJQ & $\mathrm{NEl}$ & $\begin{array}{l}60 \text { min per time, } 2 \text { times per day for } 12 \\
\text { months }\end{array}$ & (1)(2)(3) \\
\hline Zhang et al. (46) & $30 / 30$ & $\begin{array}{l}17 / 13 \\
16 / 14\end{array}$ & $\begin{array}{l}62.00 \pm 7.30 / \\
62.34 \pm 6.88\end{array}$ & $\mathrm{~N} / \mathrm{A}$ & TJQ & $\mathrm{NEl}$ & 1 time per day for 12 months & (1)(2) \\
\hline Cui et al. (47) & $28 / 28$ & $\begin{array}{l}21 / 7 \\
20 / 8\end{array}$ & $\begin{array}{l}65.80 \pm 5.20 / \\
66.50 \pm 4.80\end{array}$ & $\begin{array}{l}23.50 \pm 4.10 / \\
22.70 \pm 3.60\end{array}$ & TJQ & $\mathrm{NEl}$ & $\begin{array}{l}30 \sim 60 \text { min per time, } 2 \text { times per day for } 12 \\
\text { months }\end{array}$ & (1)(2)(3) \\
\hline Peng et al. (48) & $40 / 40$ & $\begin{array}{l}16 / 24 \\
18 / 22\end{array}$ & $\mathrm{~N} / \mathrm{A}$ & $\mathrm{N} / \mathrm{A}$ & TJQ & $\mathrm{NEl}$ & 30 min per time, 1 time per day for 6 months & (1)(2)(3) \\
\hline Liu et al. (49) & $50 / 50$ & $\begin{array}{l}30 / 20 \\
28 / 22\end{array}$ & $\begin{array}{l}53.50 \pm 6.10 / \\
53.10 \pm 5.60\end{array}$ & $\begin{array}{l}9.24 \pm 0.31 / \\
8.72 \pm 0.34\end{array}$ & TJQ & $\mathrm{NEl}$ & 60 min per time, 1 time per day for 12 months & (1)(2)(3) \\
\hline Zhang et al. (50) & $30 / 30$ & $\begin{array}{l}20 / 10 \\
19 / 11\end{array}$ & $\begin{array}{l}53.12 \pm 6.12 / \\
53.62 \pm 7.14\end{array}$ & N/A & TJQ & NEl & 8 months & (1) \\
\hline He et al. (51) & $45 / 45$ & $\mathrm{~N} / \mathrm{A}$ & $\mathrm{N} / \mathrm{A}$ & N/A & TJQ & NEl & $\begin{array}{l}45 \sim 60 \text { min per time, } 1 \text { time per day for } 1 \\
\text { month }\end{array}$ & (1)(2) \\
\hline Yao et al. (52) & $40 / 40$ & $\begin{array}{l}20 / 20 \\
25 / 15\end{array}$ & $\begin{array}{l}66.10 \pm 4.00 / \\
66.20 \pm 4.20\end{array}$ & N/A & TJQ & $\mathrm{NEl}$ & 30 min per time, 1 time per day for 3 months & (1) \\
\hline Li et al. (53) & $35 / 35$ & $\begin{array}{c}25 / 10 \\
30 / 5\end{array}$ & $\begin{array}{l}72.00 \pm 2.50 / \\
73.00 \pm 3.00\end{array}$ & $\begin{array}{l}22.00 \pm 7.00 / \\
21.00 \pm 8.00\end{array}$ & TJQ & $\mathrm{NEl}$ & 40 min per time, 1 time per day for 6 months & (1) \\
\hline Li et al. (54) & $26 / 23$ & $\begin{array}{l}19 / 7 \\
17 / 6\end{array}$ & $\begin{array}{l}65.00 \pm 2.60 / \\
66.00 \pm 2.80\end{array}$ & $\begin{array}{l}14.00 \pm 2.10 / \\
13.00 \pm 1.50\end{array}$ & TJQ & $\mathrm{NEl}$ & $\begin{array}{l}30 \text { min per time, } 5 \sim 7 \text { times per week, for } 3 \\
\text { months }\end{array}$ & (1) \\
\hline Wang et al. (55) & $26 / 24$ & $\begin{array}{l}23 / 3 \\
21 / 3\end{array}$ & $\begin{array}{l}67.83 \pm 5.32 / \\
67.86 \pm 5.98\end{array}$ & $\begin{array}{l}7.60 \pm 7.75 / \\
8.84 \pm 7.18\end{array}$ & TJQ & NEl & $\begin{array}{l}60 \text { min per time, } 3 \text { times per week, for } 3 \\
\text { months }\end{array}$ & (1)(2)(3) \\
\hline Niu et al. (56) & $20 / 20$ & $\begin{array}{l}19 / 1 \\
18 / 2\end{array}$ & $\begin{array}{l}59.70 \pm 2.76 / \\
61.30 \pm 2.89\end{array}$ & N/A & TJQ & NEl & 30 min per time, 1 time per day for 6 months & (2) \\
\hline Wei et al. (57) & $48 / 45$ & $\begin{array}{l}41 / 7 \\
36 / 9\end{array}$ & $\begin{array}{l}58.66 \pm 7.56 / \\
58.64 \pm 7.52\end{array}$ & $\mathrm{~N} / \mathrm{A}$ & WQX & NEl & 30 min per time, 1 time per day for 6 months & (1) \\
\hline Jia et al. (58) & $26 / 21$ & $\begin{array}{l}12 / 14 \\
11 / 10\end{array}$ & $\begin{array}{c}53.53 \pm 10.05 / \\
55.46 \pm 9.87\end{array}$ & $\mathrm{~N} / \mathrm{A}$ & WQX & NEl & 45 min per time, 1 time per day for 3 months & (1)(2) \\
\hline Liu et al. (59) & $50 / 50$ & $\begin{array}{l}40 / 10 \\
37 / 13\end{array}$ & $\begin{array}{l}74.24 \pm 9.10 / \\
67.72 \pm 9.26\end{array}$ & $\mathrm{~N} / \mathrm{A}$ & WQX & NEl & 45 min per time, 1 time per day for 3 months & (1)(2) \\
\hline Gao et al. (60) & $36 / 36$ & N/A & $\begin{array}{l}67.14 \pm 9.08 / \\
66.03 \pm 8.18\end{array}$ & $\begin{array}{l}13.69 \pm 5.67 / \\
14.78 \pm 9.24\end{array}$ & WQX & NEl & 30 min per time, 2 times per day for 3 months & (1)(2) \\
\hline Zhao et al. (61) & $30 / 30$ & $\begin{array}{l}20 / 10 \\
19 / 11\end{array}$ & $\begin{array}{l}58.91 \pm 5.86 / \\
56.66 \pm 6.43\end{array}$ & $\begin{array}{l}6.81 \pm 2.34 / \\
6.52 \pm 2.42\end{array}$ & WQX & NEl & $\begin{array}{l}40 \text { min per time, } 3 \text { times per week, for } 3 \\
\text { months }\end{array}$ & (1)(2) \\
\hline Zhu et al. (62) & $26 / 21$ & $\begin{array}{l}12 / 14 \\
11 / 10\end{array}$ & $\begin{array}{c}53.53 \pm 10.05 / \\
55.46 \pm 9.87\end{array}$ & $\mathrm{~N} / \mathrm{A}$ & WQX & NEl & 45 min per time, 1 time per day for 3 months & (1)(2) \\
\hline Cheng et al. (63) & $48 / 45$ & $\begin{array}{l}41 / 7 \\
36 / 9\end{array}$ & $\begin{array}{l}58.66 \pm 7.56 / \\
58.64 \pm 7.52\end{array}$ & $\mathrm{~N} / \mathrm{A}$ & WQX & $\mathrm{NEl}$ & $\begin{array}{l}30 \text { min per time, } 1 \sim 2 \text { times per day for } 6 \\
\text { months }\end{array}$ & (3) \\
\hline Zhang et al. (64) & $20 / 25$ & $\begin{array}{l}13 / 7 \\
16 / 9\end{array}$ & $\begin{array}{l}61.77 \pm 4.07 / \\
59.35 \pm 5.27\end{array}$ & $\mathrm{~N} / \mathrm{A}$ & YJJ & NEl & $\begin{array}{l}60 \text { min per time, } 4 \text { times per week, for } 6 \\
\text { months }\end{array}$ & (1)(2) \\
\hline Zhang et al. (65) & $42 / 45$ & $\begin{array}{c}33 / 9 \\
35 / 10\end{array}$ & $\begin{array}{c}64.77 \pm 11.07 / \\
62.35 \pm 9.27\end{array}$ & $\begin{array}{l}11.16 \pm 2.75 / \\
10.63 \pm 3.37\end{array}$ & YJJ & NEl & 60 min per time, 1 time per day for 6 months & (1)(2)(3) \\
\hline
\end{tabular}

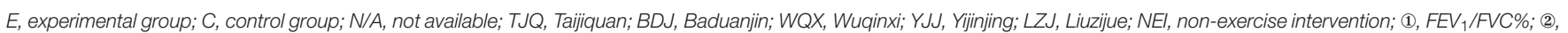
GMWT; (3), CAT. 


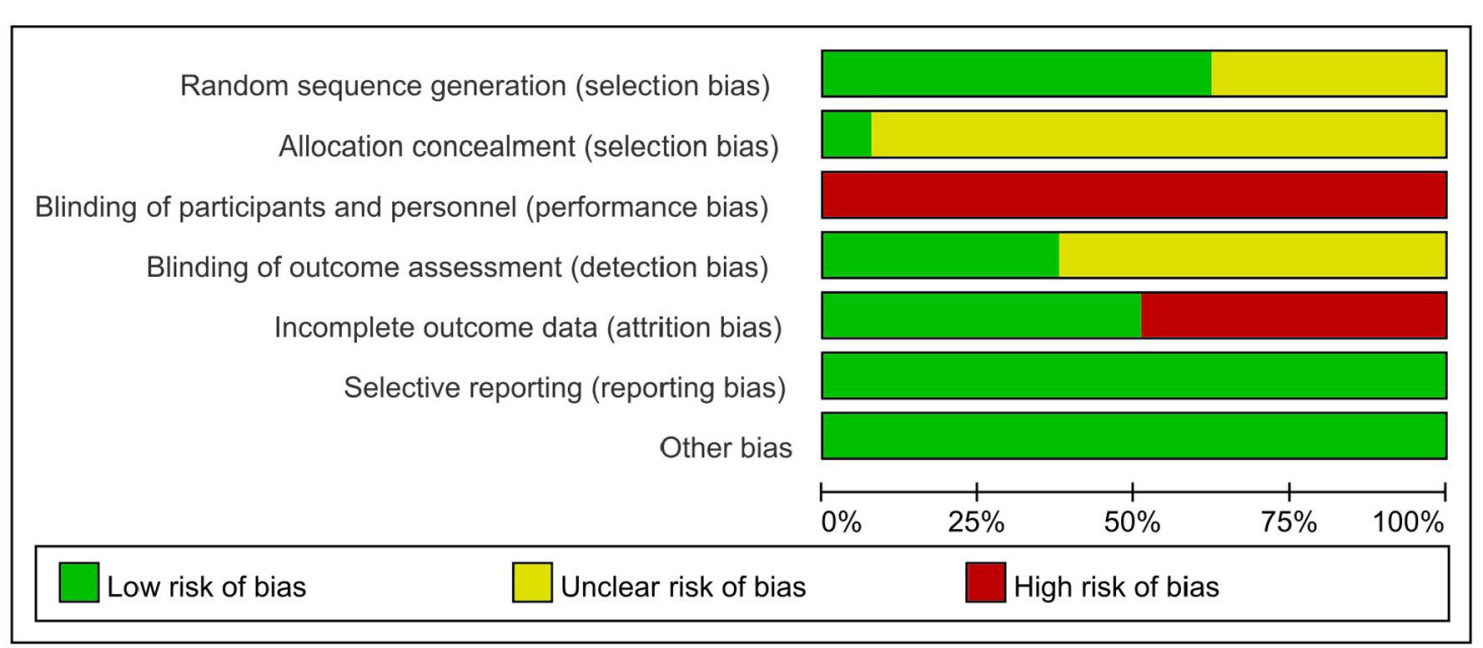

FIGURE 2 | Risk of bias assessment.

$-2.46,95 \% \mathrm{CI}-4.72$ to $-0.39, P<0.05)$; comparison of TJQ and the control group $(\mathrm{MD}=-3.35,95 \% \mathrm{CI}-5.61$ to $-1.52, P$ $<0.05)$; comparison of YJJ and the control group $(\mathrm{MD}=-8.38$, $95 \% \mathrm{CI}-13.24$ to $-3.28, P<0.05)$. The other comparisons were not statistically significant $(P>0.05)$. The probability ranking is: $\mathrm{YJJ}(P=0.86)>\mathrm{WQX}(P=0.10)>\mathrm{TJQ}(P=0.02)>\mathrm{LZJ}(P=$ $0.01)>\operatorname{BDJ}(P=0)$.

\section{Adverse Effects}

Only one study reported that patients had mild chest tightness and shortness of breath during treatment, which could be relieved after a few minutes of rest (21). Other than that, no adverse effects were reported in other studies.

\section{Publication Bias}

Inverted funnel analysis was conducted using $\mathrm{FEV}_{1} / \mathrm{FVC} \%$ as the outcome indicator, as shown in Figure 5. The inverted funnel plot is largely symmetrical, indicating no significant publication bias in this study.

\section{DISCUSSION}

Previous studies have shown that each TCE has different effects on COPD patients. Among previous studies, most reported different effects of the same TCE on different outcomes of COPD, but the comparison of different TCEs was not reported. Therefore, which TCE is most effective in alleviating COPD remains unclear.

COPD is one of the most severe public health problems globally. The prevalence rate of COPD in China is $13.7 \%$ in people over 40 years old, seriously affecting the quality of life of patients (66). At present, the TCEs promoted by the General Administration of Sport of China mainly include TJQ, BDJ, LZJ, WQX, and YJJ, which are easy to learn, well-accepted, and moderate in intensity. Based on physical, respiratory, and cardiac

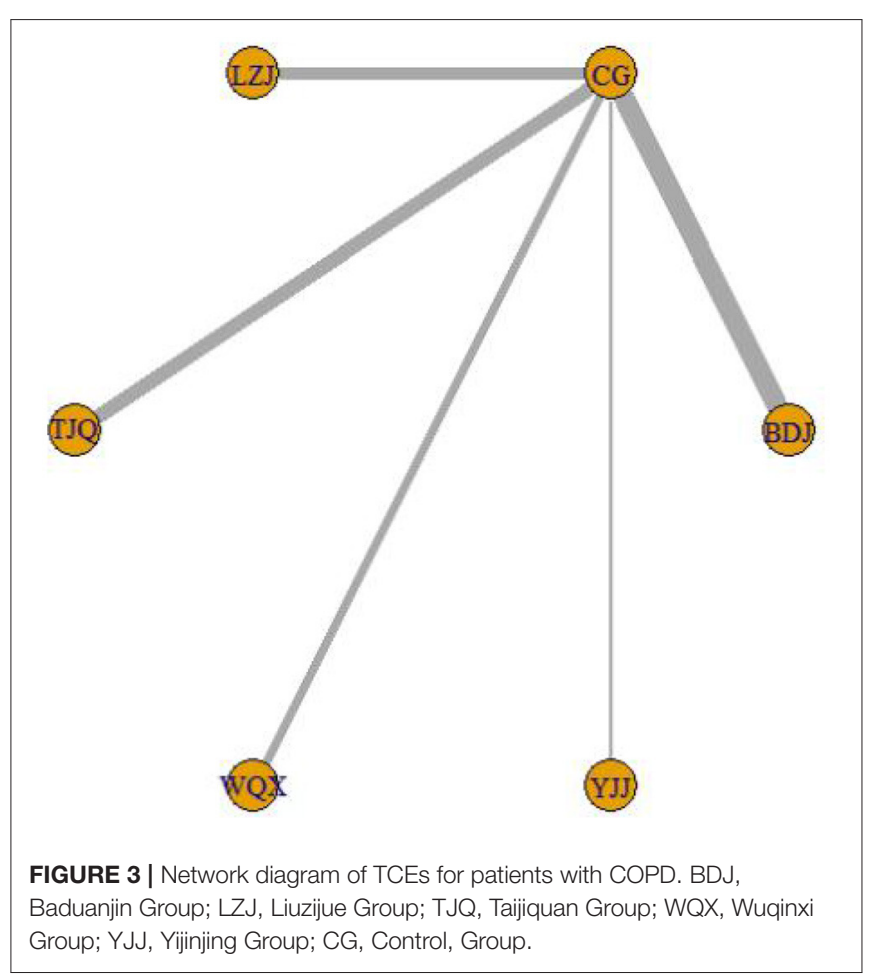

conditioning, TCEs are gradually gaining more recognition and attention as an effective treatment for COPD through endurance training, respiratory muscle training, stretch exercises, and psychological regulation, which is gradually gaining more recognition and attention $(67,68)$. In this study, the clinical efficacy of TJQ, BDJ, LZJ, WQX, and YJJ in treating COPD was objectively compared with $\mathrm{FEV}_{1} / \mathrm{FVC} \%, 6 \mathrm{MWT}$, and CAT scores as outcome indicators, providing a reliable and evidence-based medical basis for clinical treatment. 
TABLE 2 | The results of network meta-analysis.

\begin{tabular}{|c|c|c|c|c|}
\hline Intervention & & $\mathrm{FEV}_{1} / \mathrm{FVC} \%$ & 6MWT & CAT \\
\hline \multirow[t]{5}{*}{ BDJ vs } & LZJ & $-0.97(-5.33,3.49)$ & $19.29(-10.93,48.29)$ & $0.19(-3.93,4.11)$ \\
\hline & TJQ & $-0.60(-4.97,3.83)$ & $22.76(-5.41,49.81)$ & $0.88(-1.93,4.05)$ \\
\hline & WQX & $-5.15(-10.47,-0.03)^{\star}$ & $-20.79(-53.80,11.01)$ & $1.94(-3.28,7.13)$ \\
\hline & YJJ & $-0.64(-8.30,6.77)$ & $35.00(-9.35,79.87)$ & $5.92(0.23,11.20)^{\star}$ \\
\hline & $C G$ & $3.45(0.49,6.44)^{\star}$ & $53.47(36.18,71.58)^{\star}$ & $-2.45(-4.72,-0.39)^{*}$ \\
\hline \multirow{2}{*}{ LZJ vs } & YJJ & $0.30(-7.46,7.96)$ & $15.82(-30.40,62.82)$ & $5.73(-0.36,11.58)$ \\
\hline & $C G$ & $4.43(1.12,7.70)^{\star}$ & $34.32(10.44,58.78)^{\star}$ & $-2.64(-6.00,0.76)$ \\
\hline \multirow[t]{3}{*}{ TJQ vs } & WQX & $-4.58(-10.16,0.73)$ & $-43.68(-78.02,-9.40)^{\star}$ & $1.09(-4.50,5.97)$ \\
\hline & YJJ & $-0.04(-7.56,7.48)$ & $12.10(-33.21,59.23)$ & $5.03(-0.66,10.10)$ \\
\hline & CG & $4.05(0.71,7.34)^{\star}$ & $30.69(10.14,52.87)^{\star}$ & $-3.35(-5.61,-1.52)^{\star}$ \\
\hline
\end{tabular}

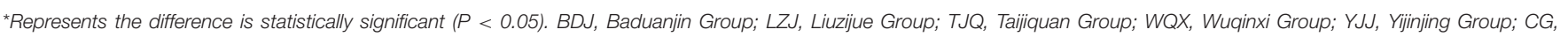
Control, Group.

TABLE 3 | Best probability ranking.

\begin{tabular}{lccc}
\hline Intervention & FEV $_{\mathbf{1}} / \mathbf{F V C} \%$ & 6MWT & CAT \\
\hline BDJ & 0.01 & 0.09 & 0.00 \\
LZJ & 0.04 & 0.01 & 0.01 \\
TJQ & 0.02 & 0.00 & 0.02 \\
WQX & 0.81 & 0.88 & 0.10 \\
YJJ & 0.12 & 0.01 & 0.86 \\
CG & 0.00 & 0.00 & 0.00 \\
\hline
\end{tabular}

BDJ, Baduanjin Group; LZJ, Liuzijue Group; TJQ, Taijiquan Group; WQX, Wuqinxi Group; YJJ, Yijinjing Group; CG, Control, Group.

Shortness of breath and breathing difficulties are two typical symptoms of COPD. In the early stages, these symptoms increase gradually when the patient feels tired, resulting in shortness of breath during daily activities or even at rest (69). Some patients with severe illness or acute exacerbations can have symptoms of wheezing and chest tightness. $\mathrm{FEV}_{1} / \mathrm{FVC} \%$ below $70 \%$ after the inhalation of bronchodilator is usually used to determine whether airflow is restricted (70). The results showed that WQX has more advantages in improving pulmonary function in patients with COPD. Due to the long duration of COPD, pulmonary function is impaired to varying degrees, resulting in breathing difficulties and restricted mobility. The limitation of activities reduces the adaptability of the body and aggravates the disease. The previous study showed that the decrease in endurance capacity is one of the main characteristics of COPD patients (71). The 6MWT score was used to evaluate the endurance capacity of the patients, with the results showing that WQX was the best intervention with the highest probability. Based on theories and practical guidance of traditional Chinese medicine, WQX incorporates the traditional movement of tigers, deers, bears, apes, and birds, effectively unblocking the meridians, harmonizing the internal organs, preventing diseases, and extending life expectancy. When performing WQX, there is no need to stick to the movement imitation. Trainees should try to embrace the charm of "Five Animals", discover the majesty of tigers, the calmness of deers, the composure of bears, the dexterity of apes, and the lightness of birds. In this way, trainees can achieve the essentials and spirit of WQX, making them feel comfortable with soft, coordinated, and symmetrical moves (72). These five movements correspond to the five internal organs of the human body. The movements of the bird are beneficial for the lungs. By stretching and lifting upper limbs, breathing can become deeper and more even, and the strength of the respiratory muscles can be enhanced. In addition, this movement can pull the lung meridians and unblock them to a certain extent, thus improving the respiratory function of the lungs (73). However, this network meta-analysis cannot determine the difference in efficacy between the single and compound interventions of WQX, and further studies are required.

As a systemic disease, COPD causes dyspnea and impaired pulmonary function by affecting the lungs. Some systemic effects caused by COPD, such as skeletal muscle dysfunction, can lead to impaired exercise ability, decreased physical activity, and decreased quality of life (74). In addition, patients with moderate or severe COPD can also develop psychiatric symptoms, including anxiety and depression (75). In terms of clinical rehabilitation, the basic goal of the treatment is to increase the self-care of patients, enable their social reintegration, and improve their quality of life. Quality of life is a comprehensive evaluation indicator reflecting the influence of disease status and treatment on physiological function, psychological function, and life of patients (76). Among different evaluation tools, the CAT scale, which is developed based on St George's respiratory disease scale, has certain reliability and validity and is used to evaluate 


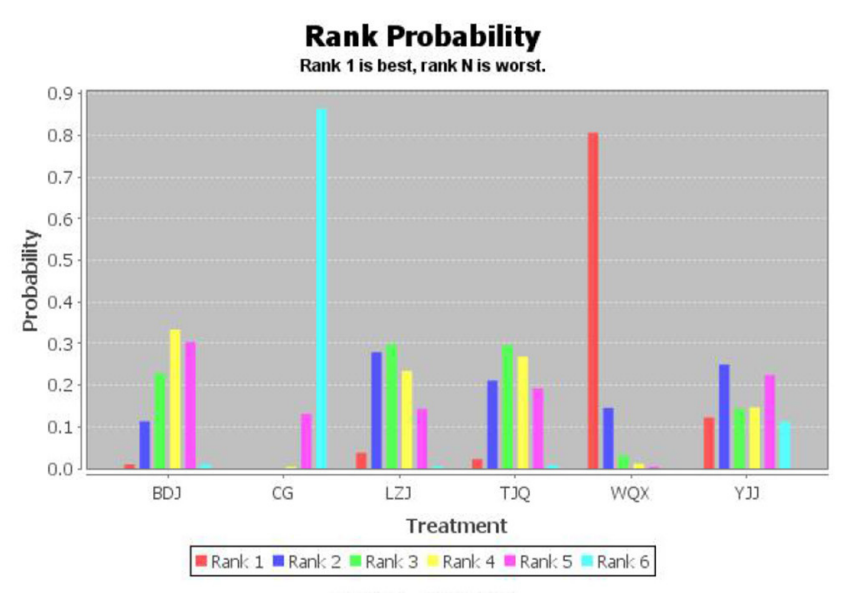

FEV1/FVC\%

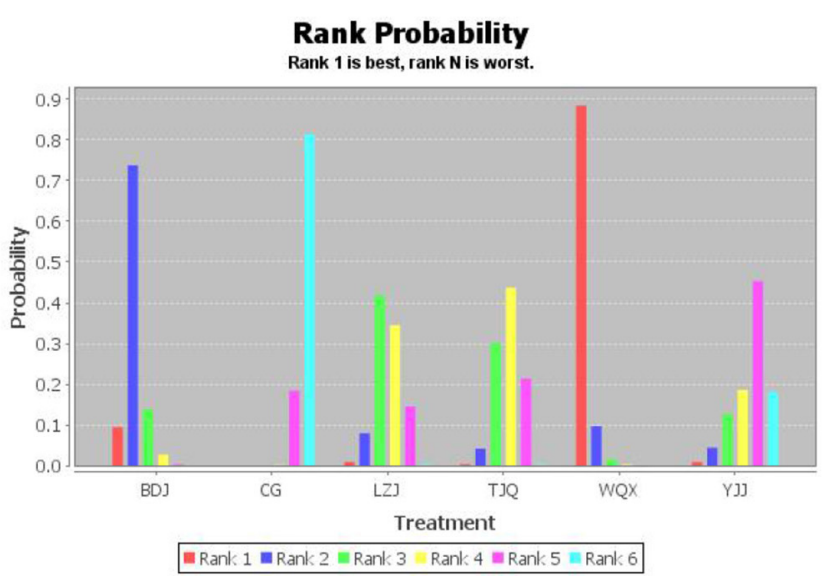

6MWT

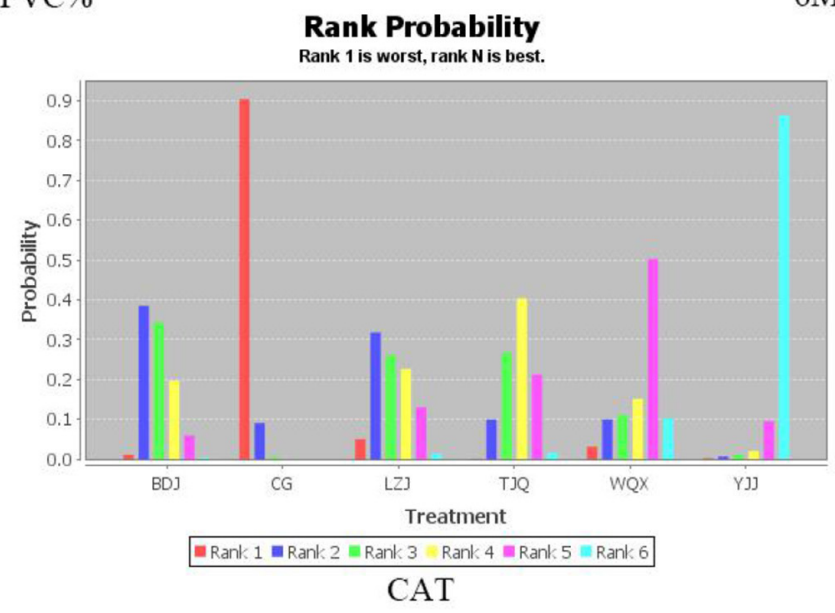

FIGURE 4 | Probability ranking of different interventions and outcome indicators. BDJ, Baduanjin Group; LZJ, Liuzijue Group; TJQ, Taijiquan Group; WQX, Wuqinxi Group; YJJ, Yijinjing Group; CG, Control, Group.

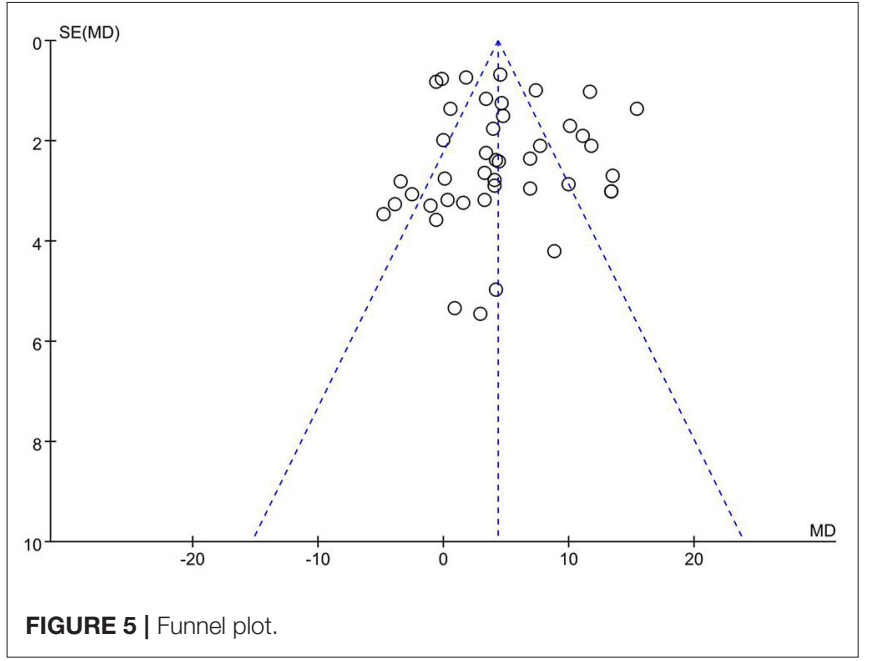

the quality of life of COPD patients (77). The results showed that YJJ has more advantages in improving the pulmonary function of COPD patients. Compared to other TCEs, YJJ emphasizes training in posture, breathing, and mind. The practices of YJJ are in accordance with 12 meridians and the Ren and Du channels of the body. Each move corresponds to the channeling of a merdian (78). Feng (79) showed that practicing YJJ for 24 weeks can increase the number of NK cells, CD4+ cells, and other immune cells. Moreover, the levels of immune factors such as IL-2 and TNF- $\alpha$ can be increased, thus delaying aging and improving the cognitive ability, mobility, and daily living skills in older people.

The American Academy of Sports Medicine recommends that adults should perform aerobic exercises at least 3-5 times a week. However, it is better for adults to perform at least 30$60 \mathrm{~min}$ of moderate-intensity exercise, 20-60 min of strenuous exercise, or a combination of moderate-intensity and strenuous exercise every day (80). The optimal exercise prescription for COPD patients is unclear (81), which could be an entry point for future studies.

Pulmonary rehabilitation has many advantages, such as improving quality of life and exercise ability, improving psychological status, and reducing morbidity and mortality. However, studies have shown that $<10 \%$ of COPD patients 
have access to specialist services $(82,83)$. This phenomenon is caused by many reasons, such as the poor medical environment in rural areas, mobilities problems of patients, and family dependency. All these problems reduce the accessibility and feasibility of pulmonary rehabilitation. Therefore, it is essential to find alternative strategies for pulmonary rehabilitation. TCEs, such as WQX and YJJ, are not limited by time and space, offering alternative methods to improve the accessibility to pulmonary rehabilitation. This result is consistent with the policy statement of the American Thoracic Society/European Respiratory Society on pulmonary rehabilitation, which states that increasing the accessibility to pulmonary rehabilitation is a key priority (84). However, most of the current studies are limited to the effects of a single TCE. Therefore, the efficacy and mechanism of different therapies should be investigated in future studies.

This network meta-analysis still has some potential bias. The 53 included RCTs (12-65) were all published in Chinese and English, which might be selectivity biased. In addition, only four RCTs $(28,48,59,64)$ employed sealed envelopes for concealed grouping, and there were no restrictions on blind methods of included studies. Moreover, the severity of the disease and the subjectivity of the outcome indicators could affect the results of the network meta-analysis. Therefore, more large-scale, doubleblind, and high-quality RCTs are needed to verify the findings in this study.

\section{CONCLUSIONS}

In conclusion, the existing evidence shows that WQX has more advantages in improving pulmonary function and endurance

\section{REFERENCES}

1. Singh D, Agusti A, Anzueto A, Barnes PJ, Bourbeau J, Celli BR, et al. Global strategy for the diagnosis, management, and prevention of chronic obstructive lung disease: the GOLD science committee report 2019. Eur Respir J. (2019) 53:1900164. doi: 10.1183/13993003.00164-2019

2. Liu S, Zhou Y, Liu S, Chen X, Zou W, Zhao D, et al. Association between exposure to ambient particulate matter and chronic obstructive pulmonary disease: results from a cross-sectional study in China. Thorax. (2017) 72:78895. doi: 10.1136/thoraxjnl-2016-208910

3. Halpin DMG, Celli BR, Criner GJ, Frith P, López Varela MV, Salvi S, et al. The GOLD Summit on chronic obstructive pulmonary disease in low- and middle-income countries. Int J Tuberc Lung Dis. (2019) 23:113141. doi: 10.5588/ijtld.19.0397

4. The GOLD Science Committee. Global Strategy for The Diagnosis, Management, and Prevention of Chronic Obstructive Pulmonary Disease (2021 Report). Available online at: https://goldcopd.org/

5. McCarthy B, Casey D, Devane D, Murphy K, Murphy E, Lacasse Y. Pulmonary rehabilitation for chronic obstructive pulmonary disease. Cochrane Database Syst Rev. (2015) CD003793. doi: 10.1002/14651858.CD003793.pub3

6. Gloeckl R, Marinov B, Pitta F. Practical recommendations for exercise training in patients with COPD. Eur Respir Rev. (2013) 22:178-86. doi: 10.1183/09059180.00000513

7. Yu Y. Network meta-analysis of four kinds of traditional Chinese fitness exercises in the treatment of osteoporosis in the elderly. China Sport Science and Technology. (2020) 56:37-44. doi: 10.16470/j.csst.202 0115

8. Huang Y, Liu J, Zhang Y, Huo C, Wen J, Luo C. Network meta-analysis of different Chinese patent medicine injections for promoting blood circulation capacity in COPD patients, while YJJ is better in improving quality of life. In addition, TCE has no significant adverse effects in treating COPD, which is recommended to be applied and promoted in clinical practices.

\section{DATA AVAILABILITY STATEMENT}

The original contributions presented in the study are included in the article/supplementary material, further inquiries can be directed to the corresponding author.

\section{AUTHOR CONTRIBUTIONS}

LL designed and wrote this study. $\mathrm{HH}$ provided guidance regarding the methodology. YY and JS reviewed the full manuscript. YW and XD took part in the data selection and extraction. YJ and $\mathrm{LH}$ performed the statistical analysis and analyzed the data. All authors contributed to the article and approved the submitted version.

\section{FUNDING}

This study was funded by Shandong Traditional Chinese Medicine Science and Technology Development Planning (No. 2017-018), Shandong University of Traditional Chinese Medicine Research and Innovation Outstanding Team (No. 220316), and Shandong Provincial Universities Scientific Research Development Planning (No. J18KB130). and resolving stasis to prevent no-reflow after PCI. Chinese Traditional and Herbal Drugs. (2021) 52:5971-83. doi: 10.7501/j.issn.0253-2670.2021. 19.020

9. Hutton B, Salanti G, Caldwell DM, Chaimani A, Schmid CH, Cameron C, et al. The PRISMA extension statement for reporting of systematic reviews incorporating network meta-analyses of health care interventions: checklist and explanations. Ann Intern Med. (2015) 162:777-84. doi: 10.7326/M1 4-2385

10. Vogelmeier CF, Criner GJ, Martinez FJ, Anzueto A, Barnes PJ, Bourbeau J, et al. Global strategy for the diagnosis, management, and prevention of chronic obstructive lung disease 2017 report. GOLD Executive Summary. Am J Respir Crit Care Med. (2017) 195:557-82. doi: 10.1164/rccm.201701-0 218PP

11. Sterne JAC, Savović J, Page MJ, Elbers RG, Blencowe NS, Boutron I, et al. RoB 2: a revised tool for assessing risk of bias in randomised trials. BMJ. (2019) 366:14898. doi: 10.1136/bmj.14898

12. Li L, Yu Y, Jia Y, Huang H. Effect of brain computer interface on upperlimb motor function after stroke: a meta-analysis. Chin J Rehabil Theory Pract. (2021) 27:765-73.

13. Deng Y, Chen J. The effects of Baduanjin's third part on rehabilitation in stable chronic obstructive pulmonary disease patients with lung and spleen deficiency syndrome. Chin J Nurs. (2015) 50:1458-63. doi: 10.376/j.issn.0254-1796.2015.12.009

14. Liang $X$. Effects of Baduanjin single lift rehabilitation exercise on rehabilitation of patients with chronic obstructive pulmonary disease. Nursing Practice and Research. (2016) 13:1567. doi: 10.3969/j.issn.1672-9676.2016.17.070

15. Lei C, Ye X, Ji W, Wang J. Effect of Baduanjin on motor endurance and quality of life in patients with stable lung and renal gas deficiency of 
chronic obstructive pulmonary disease. Chin J Rehabil Med. (2020) 35:9924. doi: 10.3969/j.issn.1001-1242.2020.08.022

16. Hou D, Huang F, Yu S, Tan Y. Luo j, Yuan Q. Study on the effect of Baduanjin exercise combined with breathing training on patients with chronic obstructive pulmonary disease in community. Guangzhou Medicine. (2017) 48:29-32. doi: 10.3969/j.issn.1000-8535.2017.06.009

17. Wu Y, Yang J. Effect of Baduanjin combined with traditional Chinese medicine on respiratory function of chronic obstructive pulmonary disease. Chin J Gerontol. (2019) 39:326-9. doi: 10.3969/j.issn.1005-9202.2019.02.025

18. Guo J, Gao Y, Xie H, Fang S, Chen G. Effect of Baduanjin exercise on patients with chronic obstructive pulmonary disease in stable stage influence of rehabilitation effect. J Qilu Nurs. (2016) 22:97-8. doi: 10.3969/j.issn.1006-7256.2016.07.051

19. Guo J. The curative effect of traditional qigong Baduanjin on chronic obstructive pulmonary disease in stable phase. Journal of Shandong Medical College. (2016) 38:171-4. doi: 10.3969/j.issn.1674-0947.2016.03.005

20. Deng W, Yang L, Dong H, Zhang W. Efficacy of deng's health care Baduanjin in pulmonary rehabilitation of patients with chronic obstructive pulmonary disease in community. Chinese Manipulation and Rehabilitation Medicine. (2020) 11:41-4. doi: 10.19787/j.issn.1008-1879.2020. 24.013

21. Zheng Y. Clinical study of respiratory Baduanjin in patients with chronic obstructive pulmonary disease (dissertation/master's thesis). Guangzhou, China: Guangzhou University of Traditional Chinese Medicine. (2019).

22. Sun Y. Clinical study on effect of health-qigong Badaunjin in treating chronic obstructive pulmonary disease (stationary phase). (dissertation/master's thesis). Changchun, China: Changchun University of Traditional Chinese Medicine. (2014).

23. Zhu Z, Chen Y. Impacts of Baduanjin qigong of sitting style on lung function in COPD patients. World J Integr Tradit Western Med. (2014) 9:846-8. doi: 10.13935/j.cnki.sjzx.140817

24. Liu S, Chen Y. Clinical study on health qigong Baduanjin for the improvement of the $6 \mathrm{~min}$ of patients with chronic obstructive pulmonary disease in stable period. Sichuan Medical Journal. (2013) 34:10902. doi: 10.16252/j.cnki.issn1004-0501-2013.08.018

25. Chen Y, Liu S, Li R, Zhang X, Zhou L, Yang G, et al. Effect of health Qigong Baduanjin on the pulmonary function of patients with chronic obstructive pulmonary disease in stable period. Chinese Medicine Modern Distance Education of China. (2015) 13:16-8. doi: 10.3969/j.issn.1672-2779.2015. 17.007

26. Huang B, Yao Q, Zhu Y. Clinical observation of health qigong Baduanjin on stable chronic obstructive pulmonary disease with lung and spleen deficiency. Hubei Journal of Traditional Chinese Medicine. (2017) 39:4-6.

27. Yu Y. Evaluation of the efficacy of fitness qigong Baduanjin in pulmonary rehabilitation in stable stage of chronic obstructive pulmonary disease. World Latest Medicine Information. (2019) 19:210-1. doi: 10.19613/j.cnki.1671-3141.2019.84.139

28. Pan M, Luo J, Yang S. Observation on rehabilitation exercise eight-section brocade in community patients with early metaphase chronic obstructive pulmonary disease. J Chengdu Univ Tradit Chin Med. (2016) 39:4952. doi: 10.13593/j.cnki.51-1501/r.2016.03.049

29. Dong L, Liu B. Effect of shaolin Baduanjin qigong on BODE index and quality of life in patients with stable chronic obstructive pulmonary disease. Clini $J$ Tradit Chin Med. (2018) 30:1465-67. doi: 10.16448/j.cjtcm.2018.0447

30. Wang L, Fang L. Effects of Baduanjin on pulmonary function in stable stage of chronic obstructive pulmonary disease. Guiding Journal of Traditional Chinese Medicine and Pharmacy. (2018) 24:86-91. doi: 10.13862/j.cnki.cn43-1446/r.2018.03.029

31. Shi J, Wang Z, Li H, Huang J, Yang P. Effect of traditional Liuzijue on lung rehabilitation. Chinese Community Doctors. (2019) 35:1803. doi: 10.3969/j.issn.1007-614x.2019.15.129

32. Zhang F. The effect of traditional Liuzijue on rehabilitation nursing of patients in COPD stable period. Guide of China Medicine. (2019) 17:248. doi: 10.15912/j.cnki.gocm.2019.01.206

33. Zheng Y. The research of rehabilitation effect on patients with COPD for lung and kidney deficiency type by treating winter disease in summer acupoint sticking therapy combined with the "six strategics" skills training. (dissertation/master's thesis). Nanjing, China: Nanjing University of Traditional Chinese Medicine. (2015).

34. Chen J, Zhang W, Zheng G, Zheng L, Li Z, Ge L. Application of Liuzijue breathing method in pulmonary rehabilitation of patients with stable COPD. Rehabilitation Medicine. (2008) 18:3-4.

35. Peng $X$. The effect of basic nursing combined with Liuzijue on the improvement of rehabilitation effect of patients in COPD stable period. Chinese Baby. (2020) 244.

36. Hou M, Cui X. The observation on the simplify six-character formula on the survival quality in qi deficiency of lung and kidney syndrome patients with COPD. Guangming Journal of Chinese Medicine. (2017) 32:351821. doi: 10.3969/j.issn.1003-8914.2017.24.007

37. Ji S, Luo G, Shi K, Yang P, Wang Z. Clinical study on sixcharacter formula in the intervention for COPD patients at stable phase. Western Journal of Traditional Chinese Medicine. (2019) 32:111-4. doi: 10.3969/j.issn.1004-6852.2019.04.031

38. Liu L. Clinical efficacy evaluation of Liuzijue respiratory exercise combined with removing phlegm and removing blood stasis soup in the treatment of stable stage of chronic obstructive pulmonary disease. (dissertation/master's thesis) Shandong, China: Shandong University of Traditional Chinese Medicine. (2018).

39. Lan Y, Han X, Wang Y, Deng W, Liu S, Feng Y. Impacts of the combined therapy of tiotropium bromide and medical exercise of the sixcharacter formula on the living quality and pulmonary function in the patients of chronic obstructive pulmonary disease at the stable stage. World Journal of Integrated Traditional and Western Medicine. (2016) 11:136995. doi: 10.13935/j.cnki.sjzx.161011

40. Guan F, Wang T, Huang Y, Chen Z, Feng L, Lin D. Effect of breathing exercises on the rehabilitation of patients with chronic obstructive pulmonary disease in stable stage. Chinese Journal of Practical Nursing. (2015) 31:211822. doi: 10.3760/cma.j.issn.1672-7088.2015.28.004

41. Li P, Liu J, Lu Y, Liu X, Wang Z, Wu W. Effects of long-term home-based Liuzijue exercise combined with clinical guidance in elderly patients with chronic obstructive pulmonary disease. Clin Interv Aging. (2018) 13:13919. doi: 10.2147/CIA.S169671

42. Xiao CM, Zhuang YC. Efficacy of Liuzijue Qigong in individuals with chronic obstructive pulmonary disease in remission. J Am Geriatr Soc. (2015) 63:14205. doi: 10.1111 /jgs. 13478

43. Wu W, Liu X, Li P, Li N, Wang Z. Effect of Liuzijue exercise combined with elastic band resistance exercise on patients with COPD: a randomized controlled trial. Evid Based Complement Alternat Med. (2018) 2018:2361962. doi: 10.1155/2018/2361962

44. Pan Y, Wang Z, Min J, Xiao W, Huang Y, Mao B, et al. Efficacy evaluation of 24-style simplified Taijiquan in pulmonary rehabilitation of stable chronic obstructive pulmonary disease. Chinese J Rehabilitation Med. (2018) 33:6816. doi: 10.3969/j.issn.1001-1242.2018.06.012

45. Zhang X, Cai Y, Zhang W, Chen X, Jia X, Zhang X, et al. Effects of twenty-four Taijiquan and respiratory function training with western medicine in patients with stable chronic obstructive pulmonary disease. J Tradit Chin Med. (2014) 55:1937-41. doi: 10.13288/j.11-2166/r.2014.22.014

46. Zhang L, Wu J, Wang Z. The effects of twenty-four Taijiquan combined with respiratory rehabilitation training on lung function and quality of life in patients with COPD. Academic Journal of Shanghai University of Traditional Chinese Medicine. (2012) 26:53-6. doi: 10.16306/j.1008-861x.2012.04.029

47. Cui C, Xing J, Yang Y, Li L. Clinical observation of noninvasive ventilator combined with Taijiquan in the treatment of stable chronic obstructive pulmonary disease. Chin Med J. (2016) 44:96-8. doi: 10.3969/j.issn.2095-8552.2016.12.034

48. Peng H, Wang P, Wang Y, Mao W, Qi C. Effect of six-movement Tai Chi rehabilitation form training on pulmonary rehabilitation of patients with chronic obstructive pulmonary disease in the stable stage. Shanghai Medical \& Pharmaceutical Journal. (2020) 41:58-62. doi: 10.3969/j.issn.1006-1533.2020.22.019

49. Liu D, Zhang Y. Effect of Taijiquan exercise on pulmonary function in middle-aged patients with chronic obstructive pulmonary disease in stable stage. Chinese Community Doctors. (2019) 35:154-7. doi: 10.3969/j.issn.1007-614x.2019.16.109 
50. Zhang S. Effect of health preserving Taijiquan on rehabilitation of chronic obstructive pulmonary disease patients. World Latest Medicine Information. (2017) 17:4-5. doi: 10.3969/j.issn.1671-3141.2017.47.002

51. He Y. The Effect of Taijiquan on lung function and ability of activity in patients with COPD stable stage. Electronic Journal Of Practical Clinical Nursing Science. (2020) 5:43-50.

52. Yao Y. Effect of Taijiquan on chronic obstructive pulmonary disease. Chinese Journal of Rehabilitation Theory and Practice. (2004) 59-60. doi: 10.3969/j.issn.1006-9771.2004. 07.028

53. Li Q, Fang W, Liu C. The effect of taijiquan combined with respiratory exercise training on rehabilitation of patients with stable chronic obstructive pulmonary disease. Chinese Journal of Rehabilitation Medicine. (2012) 27:825-8. doi: 10.3969/j.issn.1001-1242.2012. 09.009

54. Li F, Xing J, Zhang H, Zhao H, Zhang J. The influence of Taijiquan combined with respiratory training on the quality of life of COPD patients in stable period. Electronic Journal of Practical Clinical Nursing Science. (2019) 4:36-41.

55. Wang L, Wu K, Chen X, Liu Q. The Effects of Tai Chi on Lung Function, Exercise Capacity and Health Related Quality of Life for Patients With Chronic Obstructive Pulmonary Disease: A Pilot Study. Heart Lung Circ. (2019) 28:1206-12. doi: 10.1016/j.hlc.2018. 05.204

56. Niu R, He R, Luo BL, Hu C. The effect of tai chi on chronic obstructive pulmonary disease: a pilot randomised study of lung function, exercise capacity and diaphragm strength. Heart Lung Circ. (2014) 23:34752. doi: 10.1016/j.hlc.2013.10.057

57. Wei S, Cheng Y, He R. The traditional Huatuo Wuqinxi in stable patients with chronic obstructive pulmonary disease effect of pulmonary function. Clinical Journal of Traditional Chinese Medicine. (2015) 27:7935. doi: $10.16448 /$ j.cjtcm.2015.0304

58. Jia Y. The effect on chronic obstructive pulmonary disease patient in stable condition by health qigong Wuqinxi exercise early treatment. (dissertation/master's thesis). Nanjing, China: Nanjing University of Traditional Chinese Medicine. (2008).

59. Liu F, Tan P. Application of community exercise Wuqinxi in patients with chronic obstructive pulmonary disease in stable period. Journal of Henan Medical College. (2020) 32:522-4.

60. Gao Y, Qu Y, Chen M. Effect of five-animal exercises on the lung function and exercise tolerance of chronic obstructive pulmonary disease patients during discharge transition period. $J$ Clin Pathol Res. (2017) 37:975-80. doi: 10.3978/j.issn.2095-6959.2017. 05.018

61. Zhao Q. Clinical research on the prevention and cure stable phase chronic obstructive pulmonary disease in community by five-animal exercises. Acta Chinese Medicine. (2015) 30:8013. doi: $10.16368 /$ j.issn.1674-8999.2015.06.276

62. Zhu Y, Li N, Jin H. Effect on chronic obstructive pulmonary disease patient in stable condition by health qigong Wuqinxi exercise early treatment. Journal of Liaoning University of Traditional Chinese Medicin. (2010) 12:10710. doi: 10.13194/j.jlunivtcm.2010.06.109.zhuy.024

63. Chen Y, Liu S, Li R. Effect of health qigong Baduanjin on the pulmonary function of patients with chronic obstructive pulmonary disease in stable period. Clini J Tradit Chin Med. (2015) 27:6835. doi: $10.16448 /$ j.cjtcm.2015.0259

64. Zhang M, Xu G, Li F, Luo C, Meng D, Ji Y. Chinese gymnastic qigong Yijinjing promotes the function of patients with chronic obstructive pulmonary disease. Chin j Sports Med. (2016) 35:339-43. doi: 10.16038/j.1000-6710.2016. 04.005

65. Zhang M, Xv G, Luo C, Meng D, Ji Y. Qigong Yi Jinjing promotes pulmonary function, physical activity, quality of life and emotion regulation self-efficacy in patients with chronic obstructive pulmonary disease: a pilot study. J Altern Complement Med. (2016) 22:810-7. doi: 10.1089/acm. 2015.0224

66. Wang $\mathrm{C}, \mathrm{Xu} \mathrm{J}$, Yang $\mathrm{L}, \mathrm{Xu} \mathrm{Y,} \mathrm{Zhang} \mathrm{X,} \mathrm{Bai} \mathrm{C,} \mathrm{et} \mathrm{al.} \mathrm{Prevalence} \mathrm{and}$ risk factors of chronic obstructive pulmonary disease in China (the China Pulmonary Health $[\mathrm{CPH}]$ study): a national cross-sectional study. Lancet. (2018) 391:1706-17. doi: 10.1016/S0140-6736(18) 30841-9

67. Yang Y, Chen K, Tang W, Xie X, Xiao W, Xiao J, et al. Influence of Baduanjin on lung function, exercise capacity, and quality of life in patients with mild chronic obstructive pulmonary disease. Medicine (Baltimore). (2020) 99:e22134. doi: 10.1097/MD.0000000000022134

68. Ni P, Dong G, Wu X. Advances in the application of traditional Chinese exercise to lung rehabilitation in patients with chronic obstructive pulmonary disease. Guangxi Med J. (2018) 40:23324. doi: $10.11675 /$ j.issn.0253-4304.2018.19.23

69. Riley CM, Sciurba FC. Diagnosis and outpatient management of chronic obstructive pulmonary disease: a review. JAMA. (2019) 321:786-97. doi: 10.1001/jama.2019.0131

70. Bhatt SP, Balte PP, Schwartz JE, Cassano PA, Couper D, Jacobs DR Jr, et al. Discriminative accuracy of FEV1:FVC thresholds for COPD-related hospitalization and mortality. JAMA. (2019) 321:2438-47. doi: 10.1001/jama.2019.7233

71. Luo X, Zhang J, Castelberg R, Wu T, Yu P, He C, et al. The effects of traditional Chinese exercise in patients with chronic obstructive pulmonary disease: a meta-analysis. PLoS ONE. (2016) 11:e0161564. doi: 10.1371/journal.pone.0161564

72. Zhou F, Yan J, Wu J. General situation of prevention and treatment of cervical spondylosis with traditional and modern exercise therapy. Hunan Journal of Traditional Chinese Medicine. (2019) 35:164-6. doi: 10.16808/j.cnki.issn1003-7705.2019. 02.071

73. Xie L, Yin Z, Song L, Chen Y, Zhou L. Efficacy and application of Wuqinxi in pulmonary rehabilitation in stable stage of chronic obstructive pulmonary disease. Chinese Journal of Rehabilitation. (2021) 36:11720. doi: $10.3870 /$ zgkf.2021.02.012

74. Liu X, Li P, Xiao L, Lu Y, Li N, Wang Z, et al. Effects of homebased prescribed pulmonary exercise by patients with chronic obstructive pulmonary disease: study protocol for a randomized controlled trial. Trials. (2019) 20:41. doi: 10.1186/s13063-018-3149-7

75. Öztürk BÖ, Alpaydin AÖ, Özalevli S, Güler N, Cimilli C. Self-management training in chronic obstructive lung disease improves the quality of life. Turk Thorac J. (2020) 21:266-73. doi: 10.5152/TurkThoracJ.2019. 19015

76. He L. Analysis of the application of scale in health evaluation of traditional Chinese medicine. (dissertation/master's thesis) Beijing, China: Beijing University of Traditional Chinese Medicine. (2018).

77. Jones PW, Harding G, Berry P, Wiklund I, Chen WH, Kline Leidy N. Development and first validation of the COPD Assessment Test. Eur Respir J. (2009) 34:648-54. doi: 10.1183/09031936.00102509

78. Xie H. Research on the clinical application of Yi Jinjing exercise in otolaryngology. Journal of Chinese Ophthalmology and Otorhinolaryngology. (2020) 10:65-8. doi: 10.3969/j.issn.1674-9006.2020.02.002

79. Feng Y, Zhong G, Qiu W, Wei J, Zhao Z, Jing C, et al. The Effect of Fitness Qigong Yijinjing on Immunity function of the Elder. Journal of Jilin Sport University. (2013) 29:68-70.

80. Cai Q, Zhang X, Jing C, Cai S, Guo M, Li J. Effects of Elastic Resistance Exercise on Chronic Obstructive Pulmonary Disease: A Meta-analysis[J]. Chinese Journal of Rehabilitation Theory and Practice. (2021) 27:91322. doi: 10.3969/j.issn.1006?9771.2021.08.006.

81. Spruit MA, Burtin C, De Boever P, Langer D, Vogiatzis I, Wouters EF. et al. COPD and exercise: does it make a difference? Breathe (Sheff). (2016) 12:e38-49. doi: 10.1183/20734735. 003916

82. Camillo CA, Langer D, Osadnik CR, Pancini L, Demeyer H, Burtin C, et al. Survival after pulmonary rehabilitation in patients with COPD: impact of functional exercise capacity and its changes. Int $J$ Chron Obstruct Pulmon Dis. (2016) 11:2671-9. doi: 10.2147/COPD. S113450

83. da Silva MMC, Arcuri JF, Di Lorenzo VAP. Individualized, low-cost and accessible pulmonary rehabilitation program based on functional clinical tests for individuals with COPD-a study protocol of a randomized controlled trial. Trials. (2021) 22:367. doi: 10.1186/s13063-021-05267-9 
84. Rochester CL, Vogiatzis I, Holland AE, Lareau SC, Marciniuk DD, Puhan MA, et al. An official American thoracic society/European respiratory society policy statement: enhancing implementation, use, and delivery of pulmonary rehabilitation. Am J Respir Crit Care Med. (2015) 192:137386. doi: 10.1164/rccm.201510-1966ST

Conflict of Interest: The authors declare that the research was conducted in the absence of any commercial or financial relationships that could be construed as a potential conflict of interest.

Publisher's Note: All claims expressed in this article are solely those of the authors and do not necessarily represent those of their affiliated organizations, or those of the publisher, the editors and the reviewers. Any product that may be evaluated in this article, or claim that may be made by its manufacturer, is not guaranteed or endorsed by the publisher.

Copyright (c) 2022 Li, Huang, Song, Yu, Jia, Wang, Dang, Huang and Liu. This is an open-access article distributed under the terms of the Creative Commons Attribution License (CC BY). The use, distribution or reproduction in other forums is permitted, provided the original author(s) and the copyright owner(s) are credited and that the original publication in this journal is cited, in accordance with accepted academic practice. No use, distribution or reproduction is permitted which does not comply with these terms. 